\title{
OPEN Electro-osmotic flow of biological fluid in divergent channel: drug therapy in compressed capillaries
}

\author{
Yun-Jie Xu' ${ }^{1}$, Mubbashar Nazeer ${ }^{2}$, Farooq Hussain ${ }^{3}$, M. ljaz Khan ${ }^{4,5}$, M. K. Hameed ${ }^{6}$, \\ Nehad Ali Shah ${ }^{7,8}$ \& Jae Dong Chung ${ }^{7 凶}$
}

The multi-phase flow of non-Newtonian through a divergent channel is studied in this article. Jeffrey fluid is considered as the base liquid and tiny gold particles for the two-phase suspension. Application of external electric field parallel to complicated capillary with net surface charge density causes the bulk motion of the bi-phase fluid. In addition to, electro-osmotic flow with heat transfer, the simultaneous effects of viscous dissipation and nonlinear thermal radiation have also been incorporated. Finally, cumbersome mathematical manipulation yields a closed-form solution to the nonlinear differential equations. Parametric study reveals that more thermal energy is contributed in response to Brinkman number which significantly assists gold particles to more heat attain high temperature, as the remedy for compressed or swollen capillaries/arteries.

Electro-osmotic flow ${ }^{1,2}$ is mainly concerned with the application of electric field which is applied externally. Electricity/electric fields interact to charge density acting in the transverse direction, to generate the motion of bulk fluid in the direction, parallel to the applied electric fields. Nazeer et al. ${ }^{3}$ provide an analytical solution for the non-Newtonian fluid flow under the effects of applied electric fields. $\mathrm{In}^{4}$, electro-osmotic flow of two-phase Newtonian fluid is investigated through three different configurations. The study reveals that jet-shaped geometry is quite suitable for multiphase flows. Ellahi et al. ${ }^{5}$ incorporate the lubricating walls on the symmetric channels for analyzing electro-osmotic flows of a Newtonian fluid. Mekheimer et al. ${ }^{6}$ are relevant to electro-osmotic flows of two types of nanofluids suspended with gold and copper particles. Blood is used as the base fluid to form the physiological nanofluids under the effects of entropy generation. Saleem et al. ${ }^{7}$ performed the symbolic software to obtain the exact solution of the electro-osmotic flow of complex rheological fluid.

Non-Newtonian fluid has vast application in daily life from geographical flow to industrial and mechanical flow. Jeffrey fluid is also one of the non-Newtonian fluids which linearly relate relaxation time to retardation time. Firdous et al. ${ }^{8}$ discussed the simultaneous influences of magnetic fields on the Jeffrey fluid flow. To expedite the flow and heat transfer different kinds of slip conditions such as momentum slip and thermal slip boundary conditions are imposed.

An analytical prediction of multiple solutions for MHD Jeffrey-Hamel flow and heat transfer utilizing KKL nanofluid model is reported by Rana et al. ${ }^{9}$. Ahmed et al. ${ }^{10}$ consider the nanofluid flow of Jeffrey fluid, due to stretching surface with the application of external heating effects. Ellahi et al. ${ }^{11,12}$ applied separation of variables to analyze the two-dimensional flow of Jeffrey fluid through a rectangular duct. An exact solution explicitly elaborates the magnetohydrodynamics (MHD) and lubrication effects on the parallel walls on the peristaltic transport of Jeffrey fluid respectively with and without porosity.

Multi-phase flow is a ubiquitous phenomenon such as from rain to rivers flowing through valleys, from the extraction of crude oils to immiscible-liquid mixtures, from different kinds of chemical and pharmaceutical processes to biological fluid transporting in the human body, etc., are some common examples of multiphase flows ${ }^{13-17}$. Ellahi et al. ${ }^{18}$ formulate four different kinds of multiphase flows suspended with Newtonian fluids.

\footnotetext{
${ }^{1}$ School of Engineering, Huzhou University, Huzhou 313000, People's Republic of China. ${ }^{2}$ Department of Mathematics, Institute of Arts and Sciences, Government College University Faisalabad Chiniot Campus, Chiniot 35400, Pakistan. ${ }^{3}$ Department of Mathematical Sciences (FBAS), BUITEMS, Quetta 87300, Pakistan. ${ }^{4}$ Department of Mathematics and Statistics, Riphah International University, 1-14, Islamabad 44000, Pakistan. ${ }^{5}$ Nonlinear Analysis and Applied Mathematics (NAAM) Research Group, Department of Mathematics, Faculty of Science, King Abdulaziz University, P.O. Box 80257, Jidda 21589, Saudi Arabia. ${ }^{6}$ Department of Mathematics, Riphah International University Faisalabad Campus, Faisalabad 38000, Pakistan. ${ }^{7}$ Department of Mechanical Engineering, Sejong University, Seoul 05006, Korea. ${ }^{8}$ Department of Mathematics, Lahore Leads University, Lahore, Pakistan. ${ }^{\boxplus}$ email: jdchung@sejong.ac.kr
} 


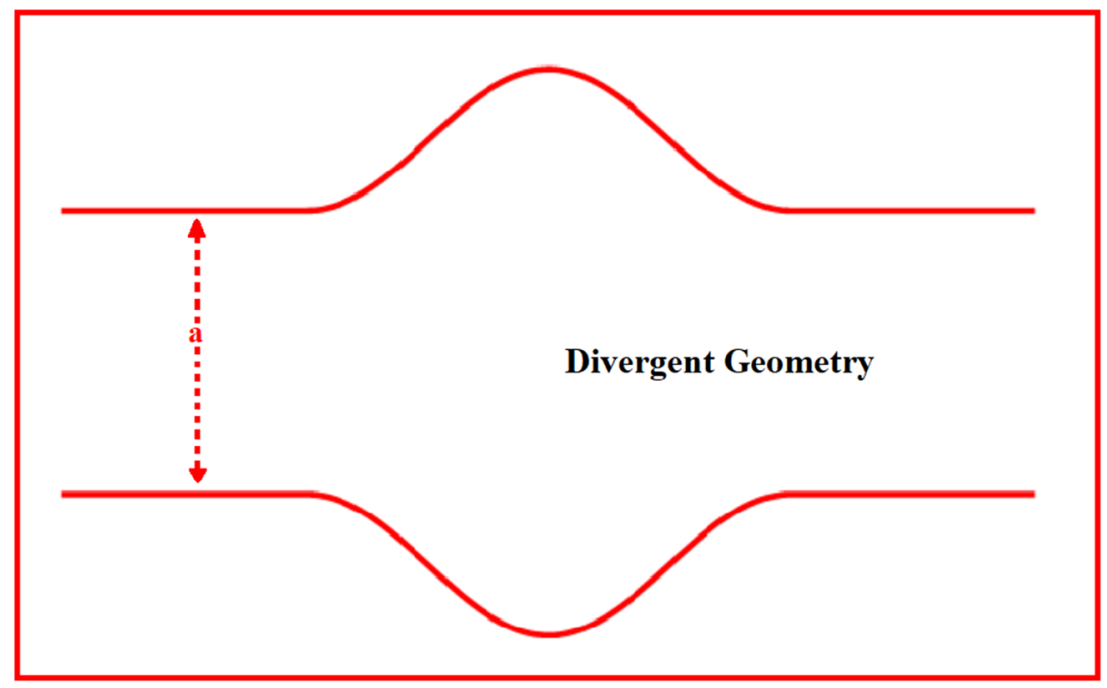

Figure 1. Divergent geometry.

They applied a thin shinning sheet/layer on the rotating disk, by using gold and silver particles. They inferred that ethanol suspension with gold particles yields a perfect coating on any rotating surface. Nazeer et al. ${ }^{19}$ obtained an approximate solution for two different kinds of multiphase flows. Suspensions are formed by considering Third-grade fluid as the base liquid while, Hafnium and crystal particles are considered. The gravitational force causes the flow of an MHD multiphase flow through an inclined channel. $\mathrm{In}^{16}$, Couette flow of Couple stress fluid is simulated. The magnetized moving upper wall of the channel drives the two-phase flow. The heating effects at the boundary attenuates the shear thickening effects. Zeeshan et al. ${ }^{20}$ have applied numerical techniques for a free-stream flow on an inclined sheet. Range-Kutta method with shooting technique is applied to obtain a numerical solution of nonlinear differential equations. Paul et al. ${ }^{21}$ worked on modeling of industrial particle and multiphase flows using combinations of DEM for free surface fluid-particle flows.

Internal flows through closed channels change the hydrodynamic structure of the flow. There can be rapid dynamical changes in the internal flows, for the uniform channels with the compressed portion or a divergent configuration. Zheng et al. ${ }^{22}$ brought convergent-divergent slit ribs to improve internal cooling. They observed that there is a vivid thermal enhancement working with small-angle trapezoidal slits that increase heat transfer. Mekheimer et al. ${ }^{23}$ use gold nanoparticles as drug agents for therapy and, suggest that gold nanoparticles effectively contribute to drug delivery. Intrauterine particle-fluid motion through a compliant asymmetric tapered channel with heat transfer is reported by Bhatti et al. ${ }^{24}$. $\mathrm{In}^{25,26}$, nano-blood flows through catharized tapered arteries are reported. Jeffrey fluid is treated as the physiological fluid by using gold nano-particles work as the remedy. Some important studies are listed in the Refs. ${ }^{27-31}$.

In view of fore-going literature, it is evident that no attention has been paid towards the two-phase flow of Jeffrey fluid with heat transfer in a convergent channel. Additional contributions of viscous dissipation and nonlinear radiative flux are taken into account, as well. The electro-osmotic flow of Jeffrey fluid suspended with gold particles is an innovative concept which addresses the blood transport through compressed/swollen capillaries or arteries.

\section{Mathematical model}

Consider a steady two-phase flow of a non-Newtonian fluid with heat transfer in a divergent channel as shown in Fig. 1. The multiphase suspension is composed of Jeffrey fluid as the base liquid suspended with tiny size confined by gold particles. Let $V_{f}=\left[u_{f}(\hat{\zeta}, \hat{\eta}), 0,0\right]$ and $V_{p}=\left[u_{p}(\hat{\zeta}, \hat{\eta}), 0,0\right]$ are the velocity profiles of liquid and particle phases, respectively.

$$
H_{G}(\widehat{\zeta})=\left\{\begin{array}{c}
a_{1}-a_{2} \sin ^{2}\left(\frac{\pi \widehat{\zeta}}{\lambda}\right) \text { When } \frac{\lambda 11}{7}<\widehat{\zeta}<\frac{\lambda 33}{7} \\
0.5 a_{1} ;
\end{array}\right.
$$

For the fluid phase,

$$
\nabla \cdot \widehat{\boldsymbol{V}}_{f}=0
$$




$$
\rho_{f}(1-\mathrm{C}) \frac{D \widehat{\boldsymbol{V}}_{f}}{D t}=-(1-\mathrm{C}) \nabla \cdot \widehat{p}+\frac{(1-\mathrm{C})}{1+\lambda_{1}} \nabla \cdot \boldsymbol{S}+S C\left(\widehat{\boldsymbol{V}}_{p}-\widehat{\boldsymbol{V}}_{f}\right)+\sigma \mathrm{B}_{o}^{2} \widehat{u}_{f}+\rho_{f} g+\nabla^{2} \Phi{\overrightarrow{E_{\widehat{\zeta}}}}_{\widehat{\zeta}} .
$$

where the stress tensor " $S$ " is defined as ${ }^{32,33}$

$$
\begin{gathered}
S=\frac{\mu_{s}}{1+\lambda_{1}}\left(\dot{r}+\lambda_{2} \ddot{r}\right), \\
\dot{\mathrm{r}}=\mathbf{L}+\mathbf{L}^{\mathrm{T}}, \\
\ddot{r}=\frac{d \dot{r}}{d t} .
\end{gathered}
$$

For the particle phase,

$$
\begin{gathered}
\nabla \cdot \widehat{V}_{p}=0, \\
\rho_{p} \mathrm{C} \frac{D \widehat{\boldsymbol{V}}_{p}}{D t}=-\mathrm{C} \nabla \cdot \widehat{p}+\mathrm{CS}\left(\widehat{\boldsymbol{V}}_{f}-\widehat{\boldsymbol{V}}_{p}\right) .
\end{gathered}
$$

The heat equation for the multiphase flow under the consideration of the thermal radiation and viscous dissipation is defined by

$$
\rho_{f}\left(C_{p}\right)_{f} \frac{D \widehat{T}}{D t}=\nabla \cdot k \nabla \widehat{T}+\mu_{s} \phi-\left(\frac{\partial q_{r \widehat{\zeta}}}{\partial \widehat{\zeta}}+\frac{\partial q_{r \widehat{\eta}}}{\partial \widehat{\eta}}\right) .
$$

The component form of Eqs. (2), (3), (7), (8) and (9) are given as

$$
\begin{aligned}
& \frac{\partial \widehat{u}_{f}}{\partial \widehat{\xi}}+\frac{\partial \widehat{v}_{f}}{\partial \widehat{\eta}}=0, \\
& \rho_{f}(1-\mathrm{C})\left[\widehat{u}_{f} \frac{\partial \widehat{u}_{f}}{\partial \widehat{\zeta}}+\widehat{v}_{f} \frac{\partial \widehat{v}_{f}}{\partial \widehat{\eta}}\right]=-(1-\mathrm{C}) \frac{\partial \widehat{p}}{\partial \widehat{\zeta}}+\frac{\mu_{s}}{1+\lambda_{1}}(1-\mathrm{C}) \\
& \left.\left[\frac{\partial^{2} \widehat{u}_{f}}{\partial \hat{\zeta}^{2}}+\frac{\partial^{2} \widehat{v}_{f}}{\partial \widehat{\eta}^{2}}\right]+\operatorname{CS}\left(\widehat{u}_{p}-\widehat{u}_{f}\right)-\sigma \mathrm{B}_{o}^{2} \widehat{u}_{f}+\left[\frac{\partial^{2} \Phi}{\partial \widehat{\zeta}^{2}}+\frac{\partial^{2} \Phi}{\partial \widehat{\eta}^{2}}\right] \vec{E}_{\widehat{\zeta}}\right\} \text {, } \\
& \frac{\partial \widehat{u}_{p}}{\partial \widehat{\zeta}}+\frac{\partial \widehat{v}_{p}}{\partial \widehat{\eta}^{\prime}}=0 \\
& \rho_{p} \mathrm{C}\left[\hat{u}_{p} \frac{\partial \widehat{u}_{p}}{\partial \widehat{\zeta}}+\widehat{v}_{p} \frac{\partial \widehat{v}_{p}}{\partial \hat{\eta}}\right]=-\mathrm{C} \frac{\partial \widehat{p}}{\partial \bar{\zeta}}+\mathrm{CS}\left(\widehat{u}_{f}-\widehat{u}_{p}\right), \\
& \rho_{f} C_{p}\left(\frac{\partial}{\partial t}+\frac{\partial}{\partial \hat{\zeta}}+\frac{\partial}{\partial \hat{\eta}}\right) \widehat{T}=\frac{\partial}{\partial \hat{\eta}}\left(k \frac{\partial \widehat{T}}{\partial \hat{\eta}}\right)+\frac{\mu_{s}}{1-\lambda_{1}}\left(\frac{\partial u}{\partial \hat{\eta}}\right)^{2}-\frac{16 \sigma^{*}}{3 k^{*}}\left(\frac{\partial^{2} \widehat{T}}{\partial \widehat{\zeta}^{2}}+\frac{\partial^{2} \widehat{T}}{\partial \hat{\eta}^{2}}\right) .
\end{aligned}
$$

The dimensional form of the boundary are defined by

$$
\begin{gathered}
\widehat{u}_{f}=\widehat{u}_{f_{\text {At the wall }}} ; \quad \text { When } \widehat{\eta}=H_{G}(\widehat{\zeta}), \\
\widehat{u}_{f}=\widehat{u}_{f_{\text {At the wall }}} ; \quad \text { When } \hat{\eta}=-H_{G}(\widehat{\zeta}) .
\end{gathered}
$$

The following transformation is used to get the non-dimensional form of the above equations 


$$
\left.\begin{array}{l}
\bar{\xi}=\frac{\widehat{\zeta}}{\lambda}, \bar{\eta}=\frac{\widehat{\eta}}{a_{1}}, \overline{\mathrm{u}}_{f}=\frac{\widehat{u}_{f}}{U_{o}}, \overline{\mathrm{u}}_{p}=\frac{\widehat{u}_{p}}{U_{o}}, \bar{v}_{f}=\frac{\widehat{v}_{f}}{\delta U_{o}}, \bar{v}_{p}=\frac{\widehat{v}_{p}}{\delta U_{o}}, \bar{h}=\frac{H}{a_{1}}, \quad \beta=\frac{a_{2}}{a_{1}}, \\
\bar{u}=\frac{\widehat{u}}{u_{o}}, \quad \bar{\mu}_{s}=\frac{\mu_{s}}{\mu_{o}}, \quad \bar{p}=\frac{a \delta \widehat{p}}{U_{o} \mu_{s}}, \bar{\Phi}=\frac{\widehat{\Phi}}{\widehat{\zeta}}, \bar{\psi}=\frac{\widehat{\psi}}{a_{1} U_{o}}, \delta=\frac{a_{1}}{\lambda}, U_{H S}=\frac{-\varepsilon \widehat{\zeta} \vec{\xi}}{U_{o} \mu_{s}}, \\
m=a_{1} e z \sqrt{\frac{2 n_{o}}{\varepsilon B_{o} T^{\prime}}}, \mathrm{M}=a_{1} B_{o} \sqrt{\frac{\sigma}{\mu_{s}}}, \quad B_{r}=\frac{\mu_{o} u^{* 2}}{k\left(T_{1}-T_{o}\right)}, \quad R_{d}=\frac{16 \sigma^{*}}{3 k^{*}}, \quad \bar{T}^{*}=\frac{\widehat{T}-T_{o}}{T_{1}-T_{o}} .
\end{array}\right\}
$$

By using the Eq. (17) into Eqs. (10)-(16), we get the following form of the dimensionless problem

$$
\begin{gathered}
\frac{\partial \mathrm{u}_{f}}{\partial \zeta}+\frac{\partial v_{f}}{\partial \eta}=0 \\
\frac{\partial(p)}{\partial(\zeta)}=\frac{1}{1+\lambda_{1}} \cdot \frac{\partial^{2} \mathrm{u}_{f}}{\partial \eta^{2}}+\frac{\mathrm{CS}\left(\mathrm{u}_{p}-\mathrm{u}_{f}\right) a^{2}}{(1-\mathrm{C}) \mu_{s}}-\left(\frac{M^{2}}{(1-\mathrm{C})}\right) \mathrm{u}_{f}-\frac{\Phi}{\varepsilon} \cdot \frac{m^{2} \mathrm{U}_{H S}}{(1-\mathrm{C})} \\
\frac{\partial \mathrm{u}_{p}}{\partial \zeta}+\frac{\partial v_{p}}{\partial \eta}=0 \\
\frac{\mu_{s}}{a \delta \lambda} \frac{\partial p}{\partial \zeta}=S\left(\mathrm{u}_{f}-\mathrm{u}_{p}\right) \\
\left(1-R_{d}\right) \frac{\partial^{2} T^{*}}{\partial \eta^{2}}+B_{r}\left(\frac{\mu_{s}}{1-\lambda_{1}}\left(\frac{\partial u}{\partial \eta}\right)^{2}\right)=0
\end{gathered}
$$

(i)

$$
u_{f}=0 ; \quad \text { When } \eta=h_{g}(\zeta)
$$

$$
\text { (ii) } \quad u_{f}=0 ; \quad \text { When } \eta=-h_{g}(\bar{\zeta})
$$

The dimensionless form of Eq. (1) is

$$
h_{g}(\zeta)= \begin{cases}1-\beta \sin ^{2}(\pi \zeta) & \text { When } 0.5<\zeta<1.5 \\ 0.5 ; & \text { Othwewise. }\end{cases}
$$

After basic manipulation, the electro-osmotic potential function $\Phi(\eta)$, given in Eq. (19), can be obtained as

$$
\Phi(\eta)=\frac{\cosh (m \eta)}{\cosh \left(m h_{g}\right)} .
$$

Using Eq. (26) in Eq. (19), we have

$$
\frac{1}{1+\lambda_{1}} \cdot \frac{\partial^{2} \mathrm{u}_{f}}{\partial \eta^{2}}-\left(\frac{M^{2}}{(1-\mathrm{C})}\right) \mathrm{u}_{f}+\frac{a^{2} \mathrm{CS}\left(\mathrm{u}_{p}-\mathrm{u}_{f}\right)}{(1-\mathrm{C}) \mu_{s}}-\left(\frac{\mathrm{m}^{2} \mathrm{U}_{H S}}{(1-\mathrm{C})}\right) \frac{\cosh (m \eta)}{\cosh \left(m h_{g}\right)}=\frac{\partial p}{\partial \zeta} .
$$

From Eq. (21), we can write

$$
\mathrm{u}_{p}=\mathrm{u}_{f}-\left(\frac{\mu_{s}}{a \delta \lambda S}\right) \frac{\partial p}{\partial \zeta} .
$$

Substituting Eq. (28) into Eq. (27), we get

$$
\frac{\partial^{2} \mathrm{u}_{f}}{\partial \eta^{2}}-A_{1} \mathrm{u}_{f}=A_{2} P+A_{3} \cosh (m \eta)
$$

By solving the above equation, we get the following form of required quantities 


$$
\begin{aligned}
& u_{f}=\left(-\frac{P\left(1+\lambda_{1}\right) \pi_{d}+\left(\left(1+\lambda_{1}\right) \cosh \left[h_{g} m\right]+m \beta \sinh \left[h_{g} m\right]\right) \pi_{e}}{2\left(\left(1+\lambda_{1}\right) \cosh \left[h_{g} \sqrt{\pi_{a}}\right]+\beta \sinh \left[h_{g} \sqrt{\pi_{a}}\right] \sqrt{\pi_{a}}\right)}\right) \\
& \times\left(\cosh \left[\sqrt{\pi_{a}} \eta\right]+\sinh \left[\sqrt{\pi_{a}} \eta\right]\right)+\left(\cosh \left[\sqrt{\pi_{a}} \eta\right]-\sinh \left[\sqrt{\pi_{a}} \eta\right]\right) \\
& \left.\times\left(-\frac{P\left(1+\lambda_{1}\right) \pi_{d}+\left(\left(1+\lambda_{1}\right) \cosh \left[h_{g} m\right]+m \beta \sinh \left[h_{g} m\right]\right) \pi_{e}}{2\left(\left(1+\lambda_{1}\right) \cosh \left[h_{g} \sqrt{\pi_{a}}\right]+\beta \sinh \left[h_{g} \sqrt{\pi_{a}}\right] \sqrt{\pi_{a}}\right)}\right)+\pi_{d} P+\pi_{e} \cosh [m \eta] .\right) \\
& u_{p}=\left(-\frac{P\left(1+\lambda_{1}\right) \pi_{d}+\left(\left(1+\lambda_{1}\right) \cosh \left[h_{g} m\right]+m \beta \sinh \left[h_{g} m\right]\right) \pi_{e}}{2\left(\left(1+\lambda_{1}\right) \cosh \left[h_{g} \sqrt{\pi_{a}}\right]+\beta \sinh \left[h_{g} \sqrt{\pi_{a}}\right] \sqrt{\pi_{a}}\right)}\right) \\
& \times\left(\cosh \left[\sqrt{\pi_{a}} \eta\right]+\sinh \left[\sqrt{\pi_{a}} \eta\right]\right)+\left(\cosh \left[\sqrt{\pi_{a}} \eta\right]-\sinh \left[\sqrt{\pi_{a}} \eta\right]\right) \\
& \times\left(-\frac{P\left(1+\lambda_{1}\right) \pi_{d}+\left(\left(1+\lambda_{1}\right) \cosh \left[h_{g} m\right]+m \beta \sinh \left[h_{g} m\right]\right) \pi_{e}}{2\left(\left(1+\lambda_{1}\right) \cosh \left[h_{g} \sqrt{\pi_{a}}\right]+\beta \sinh \left[h_{g} \sqrt{\pi_{a}}\right] \sqrt{\pi_{a}}\right)}\right) \\
& +\pi_{d} P+\pi_{e} \cosh [m \eta]-\left(\frac{\mu_{s}}{a \delta \lambda S}\right) P \text {. } \\
& T=c_{1}+\eta c_{2}+\frac{1}{4\left(1+R_{d}\right)\left(m^{2}-\pi_{a}\right)\left(1+\lambda_{1}\right)} B_{r} \\
& \left(\begin{array}{l}
-\frac{1}{2} m^{2}\left(R_{3}^{2} \cosh \left[2 \eta \sqrt{\pi_{a}}\right]+R_{4}^{2} \cosh \left[2 \eta \sqrt{\pi_{a}}\right]+2 R_{3} R_{4} \sinh \left[2 \eta \sqrt{\pi_{a}}\right]\right)+\frac{1}{2} \\
\left(R_{3}^{2}+R_{4}^{2}\right) \cosh \left[2 \eta \sqrt{\pi_{a}}\right] \pi_{a}+R_{3} R_{4} \sinh \left[2 \eta \sqrt{\pi_{a}}\right] \pi_{a}-\left(R_{3}^{2}-R_{4}^{2}\right) \eta^{2} \pi_{a}^{2} \\
+\frac{8 m^{3} \sinh [m \eta]\left(R_{4} \cosh \left[\eta \sqrt{\pi_{a}}\right]+R_{3} \sinh \left[\eta \sqrt{\pi_{a}}\right]\right) \sqrt{\pi_{a}} \pi_{e}}{\left(-m+\sqrt{\pi_{a}}\right)\left(m+\sqrt{\pi_{a}}\right)} \\
-\frac{16 m^{2} \cosh [m \eta]\left(R_{3} \cosh \left[\eta \sqrt{\pi_{a}}\right]+R_{4} \sinh \left[\eta \sqrt{\pi_{a}}\right]\right) \pi_{a} \pi_{e}}{\left(-m+\sqrt{\pi_{a}}\right)\left(m+\sqrt{\pi_{a}}\right)} \\
+\frac{8 m \sinh [m \eta]\left(R_{4} \cosh \left[\eta \sqrt{\pi_{a}}\right]+R_{3} \sinh \left[\eta \sqrt{\pi_{a}}\right]\right) \pi_{a}^{3 / 2} \pi_{e}}{\left(-m+\sqrt{\pi_{a}}\right)\left(m+\sqrt{\pi_{a}}\right)}+m^{4} \eta^{2} \pi_{a}^{2} \\
-\frac{1}{2} m^{2} \cosh [2 m \eta] \pi_{e}^{2}+\frac{1}{2} \cosh [2 m \eta] \pi_{a} \pi_{e}^{2}+m^{2} \eta^{2} \pi_{a}\left(R_{3}^{2}-R_{4}^{2}-\pi_{e}^{2}\right)
\end{array}\right) \mu_{s} .
\end{aligned}
$$

The volumetric flow rate is

$$
\begin{aligned}
& Q=\left(\begin{array}{l}
P\left(h_{g}\left(1+\lambda_{1}\right) \cosh \left[h \sqrt{\pi_{a}}\right]+\frac{\sinh \left[h_{g} \sqrt{\pi_{a}}\right]\left(-1-\lambda_{1}+h_{g} \beta \pi_{a}\right)}{\sqrt{\pi_{a}}}\right) \pi_{e} \\
\left(\begin{array}{l}
-\sinh \left[h_{g} \sqrt{\pi_{a}}\right]\left(m\left(1+\lambda_{1}\right) \cosh \left[h_{g} m\right]+\beta \sinh \left[h_{g} m\right]\left(m^{2}-\pi_{a}\right)\right) \\
+\left(1+\lambda_{1}\right) \cosh \left[h_{g} \sqrt{\pi_{a}}\right] \sinh \left[h_{g} m\right] \sqrt{\pi_{a}}
\end{array}\right) \pi_{e} \\
m \sqrt{\pi_{a}}
\end{array}\right) \\
& /\left(\left(1+\lambda_{1}\right) \cosh \left[h_{g} \sqrt{\pi_{a}}\right]+\beta \sinh \left[h_{g} \sqrt{\pi_{a}}\right] \sqrt{\pi_{a}}\right)+h_{g} P \pi_{d}-\frac{P \sinh \left[h_{g} \sqrt{\pi_{a}}\right] \pi_{a}}{\left(1+\lambda_{1}\right) \cosh \left[h_{g} \sqrt{\pi_{a}}\right] \sqrt{\pi_{a}}} \\
& +\beta \sinh \left[h_{g} \sqrt{\pi_{a}}\right] \pi_{a} \\
& -\frac{P \lambda_{1} \sinh \left[h_{g} \sqrt{\pi_{a}}\right] \pi_{d}}{\left(1+\lambda_{1}\right) \cosh \left[h_{g} \sqrt{\pi_{a}}\right] \sqrt{\pi_{a}}+\beta \sinh \left[h_{g} \sqrt{\pi_{a}}\right] \pi_{a}}+\frac{\sinh \left[h_{g} m\right] \pi_{e}}{m} \\
& \cosh \left[h_{g} m\right] \pi_{e} \\
& -\overline{\left(1+\lambda_{1}\right) \operatorname{coth}\left[h_{g} \sqrt{\pi_{a}}\right] \sqrt{\pi_{a}}+\beta \pi_{a}} \\
& -\frac{\lambda_{1} \cosh \left[h_{g} m\right] \pi_{e}}{\left(1+\lambda_{1}\right) \operatorname{coth}\left[h_{g} \sqrt{\pi_{a}}\right] \sqrt{\pi_{a}}+\beta \pi_{a}}-\frac{m \beta \sinh \left[h_{g} m\right] \pi_{e}}{\left(1+\lambda_{1}\right) \operatorname{coth}\left[h_{g} \sqrt{\pi_{a}}\right] \sqrt{\pi_{a}}+\beta \pi_{a}}-\frac{h_{g} P \mu_{s}}{a S \delta \lambda} .
\end{aligned}
$$

The pressure is obtained from the above equation which is given by 


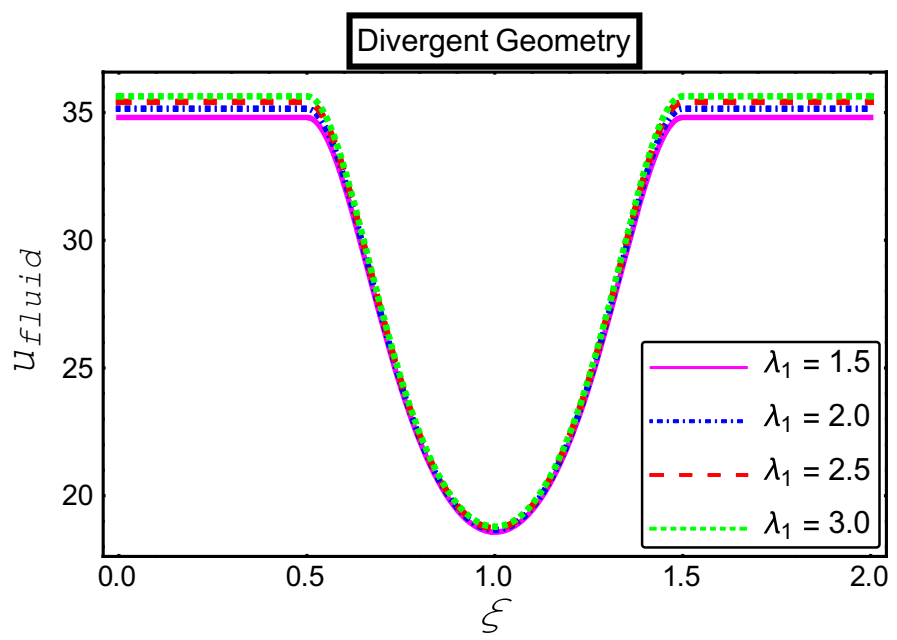

Figure 2. Impact of Jeffrey parameters on fluid velocity.

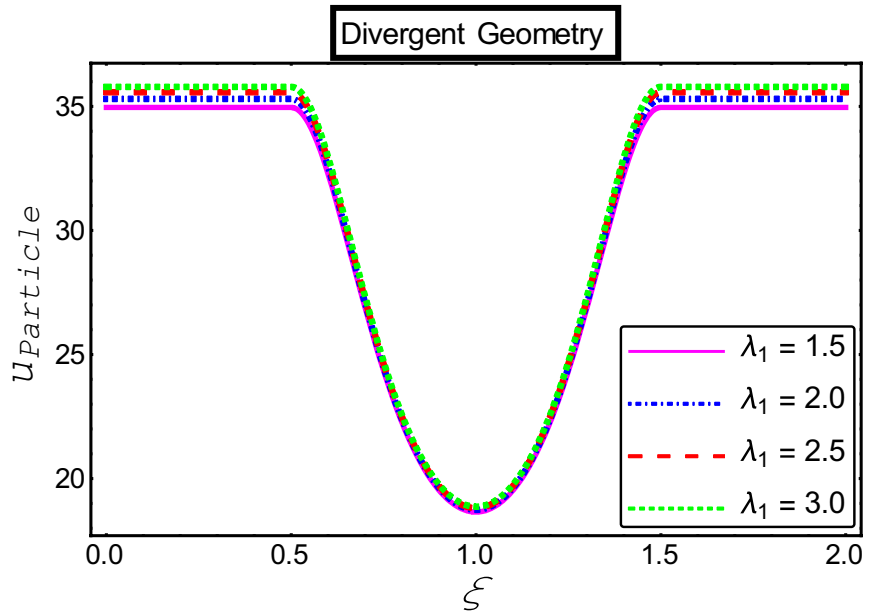

Figure 3. Impact of Jeffrey parameters on particle velocity.

$$
\left.P=\frac{\left(\operatorname{aS\delta } \lambda\left(\begin{array}{l}
2 m\left(\left(1+\lambda_{1}\right) \cosh \left[h_{g} m\right]+m \beta \sinh \left[h_{g} m\right]\right) \pi_{e} \\
+\left(1+\lambda_{1}\right) \operatorname{coth}\left[h_{g} \sqrt{\pi_{a}}\right] \sqrt{\pi_{a}}\left(m Q-2 \sinh \left[h_{g} m\right] \pi_{e}\right) \\
+\beta \pi_{a}\left(m Q-2 \sinh \left[h_{g} m\right] \pi_{e}\right)
\end{array}\right)\right.}{\left(\begin{array}{l}
2 a m S \delta \lambda\left(-1-\lambda_{1}+h_{g}\left(1+\lambda_{1}\right) \operatorname{coth}\left[h_{g} \sqrt{\pi_{a}}\right] \sqrt{\pi_{a}}+h_{g} \beta \pi_{a}\right) \pi_{d} \\
-h_{g} m\left(\left(1+\lambda_{1}\right) \operatorname{coth}\left[h_{g} \sqrt{\pi_{a}}\right]+\beta \sqrt{\pi_{a}}\right) \sqrt{\pi_{a}} \mu_{s}
\end{array}\right)}\right\}
$$

\section{Results and discussion}

The objective of this section is to highlight the impact of important physical parameters on the velocity and temperature distribution through graphs. Figures 2, 3, 4, 5, 6, 7, 8, 9, 10, 11, 12, 13, 14, 15, 16 and 17 depict the influence of most significant parameters such as Jeffrey fluid parameter $\lambda_{1}$, electro-osmotic parameter $m$, Hartmann number $M$, the concentration of particles $C$, Helmholtz-Smoluchowski velocity $U_{H S}$, and Brinkman number $B_{r}$. Figures 2, 3 and 4 provide the influence of Jeffrey fluid parameter $\lambda_{1}$ on momentum and thermal profiles for an admissible range of the parameter. A vivid reduction in heat transfer is observed, in response to variation in the parameter. This is an opposite trend to the momentum profile of each phase. The effect of $m$ on the velocity and temperature profiles is given in Figs. 4, 6 and 7. Unlike, the previous case velocity of each phase reduces while more thermal energy incorporates into the system by expediting the heat transfer. This phenomenon is the application of the electro-osmotic process which ionizes the charged groups on the surface or due to preferential adsorption of ions within the fluid. It acts transversely to the motion of the fluid due to Lorentz's force. Hartman number $M$ is a dimensionless number that corresponds to magnetic field induction. Figures 8,9 and 10 highlights 


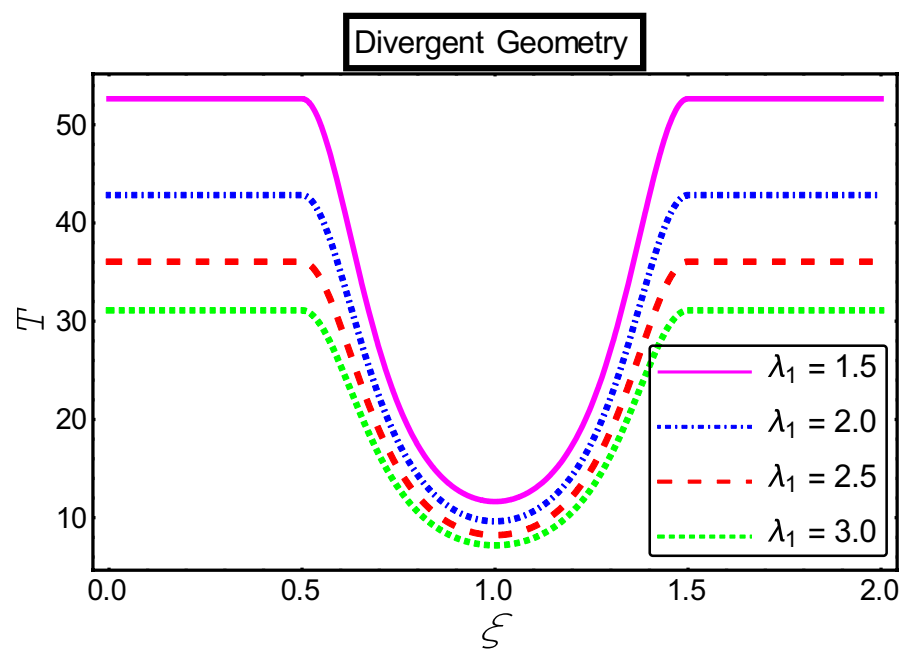

Figure 4. Impact of Jeffrey parameters on the temperature profile.

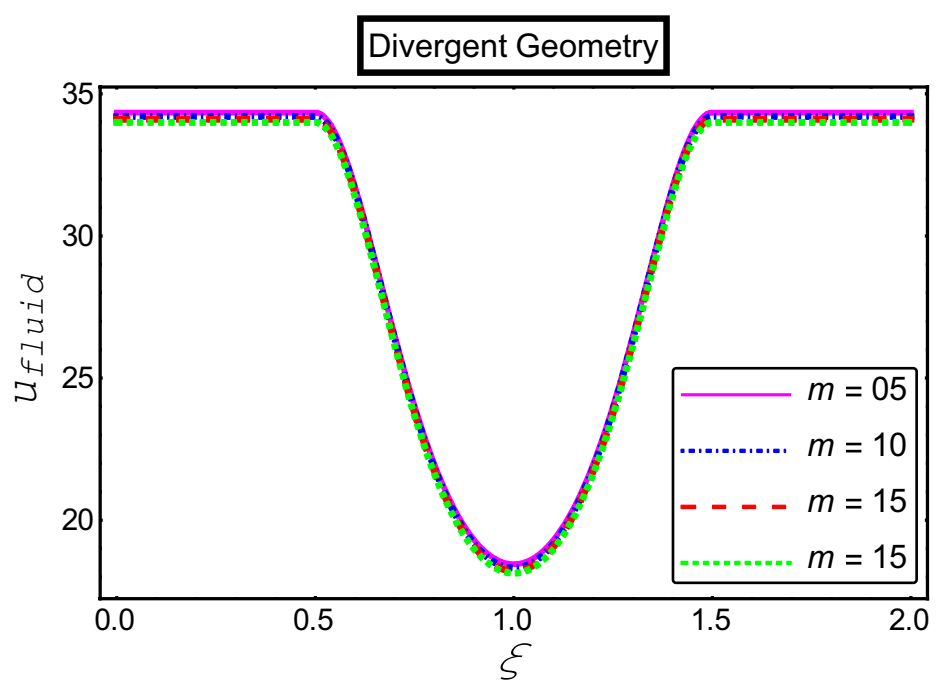

Figure 5. Impact of electro-osmotic parameter on fluid velocity.

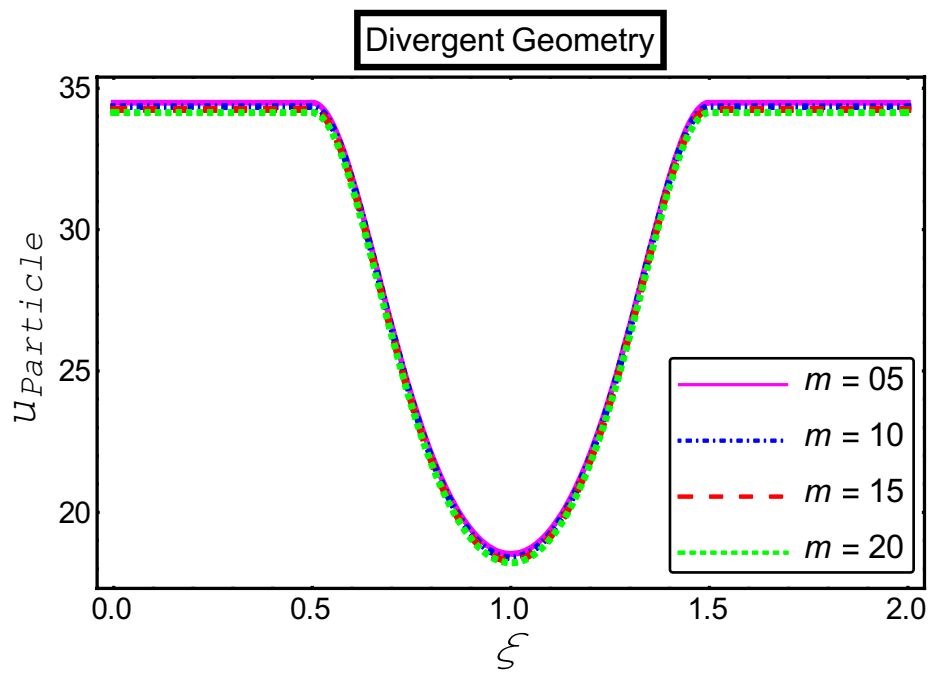

Figure 6. Impact of electro-osmotic parameter on the particle velocity. 


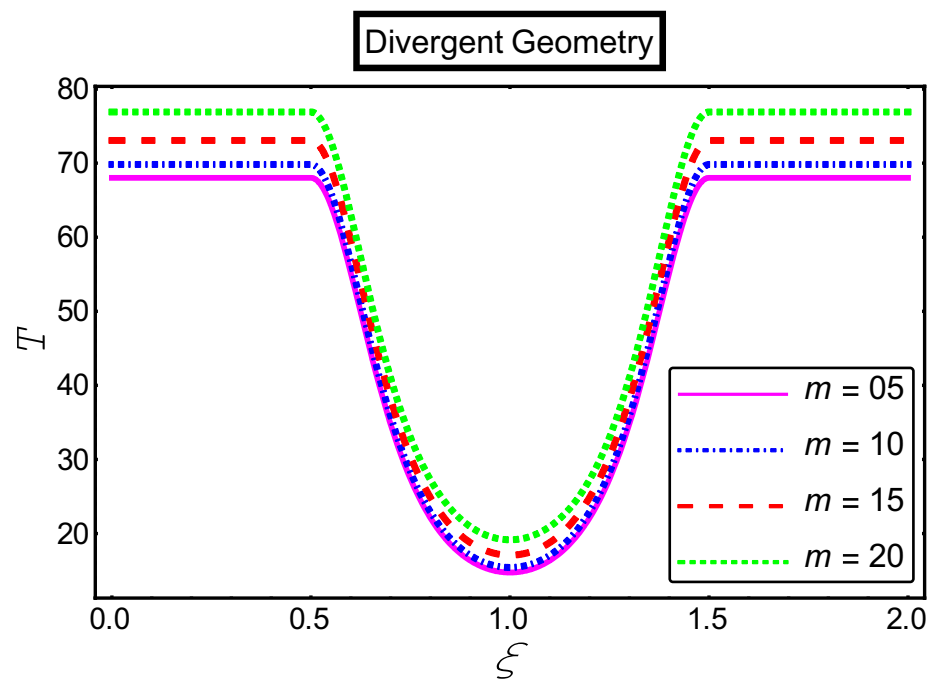

Figure 7. Impact of electro-osmotic parameter on the temperature profile.

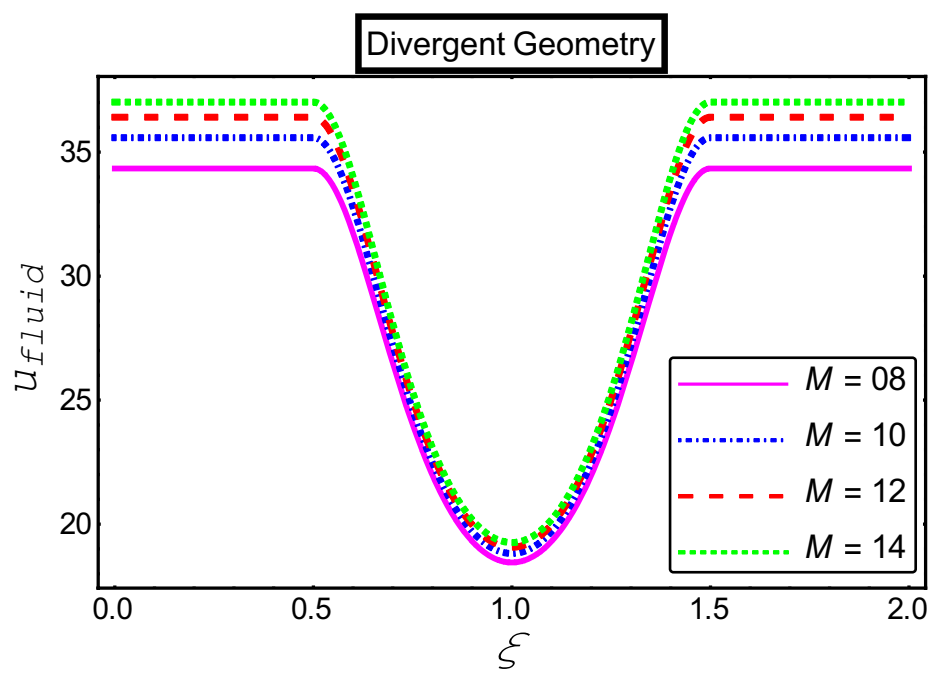

Figure 8. Impact of Hartmann numbers on fluid velocity.

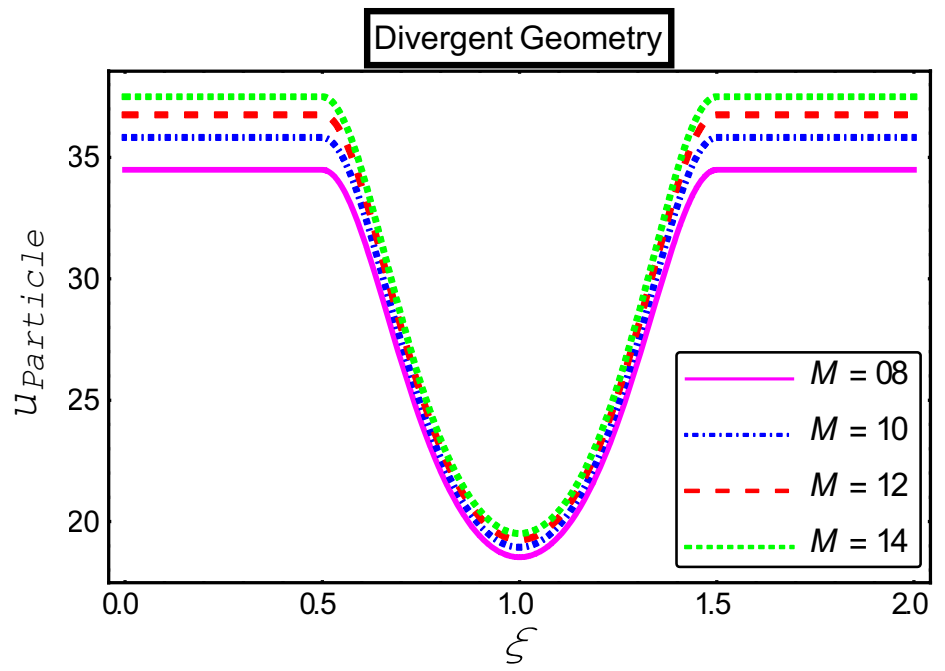

Figure 9. Impact of Hartmann numbers on particle velocity. 


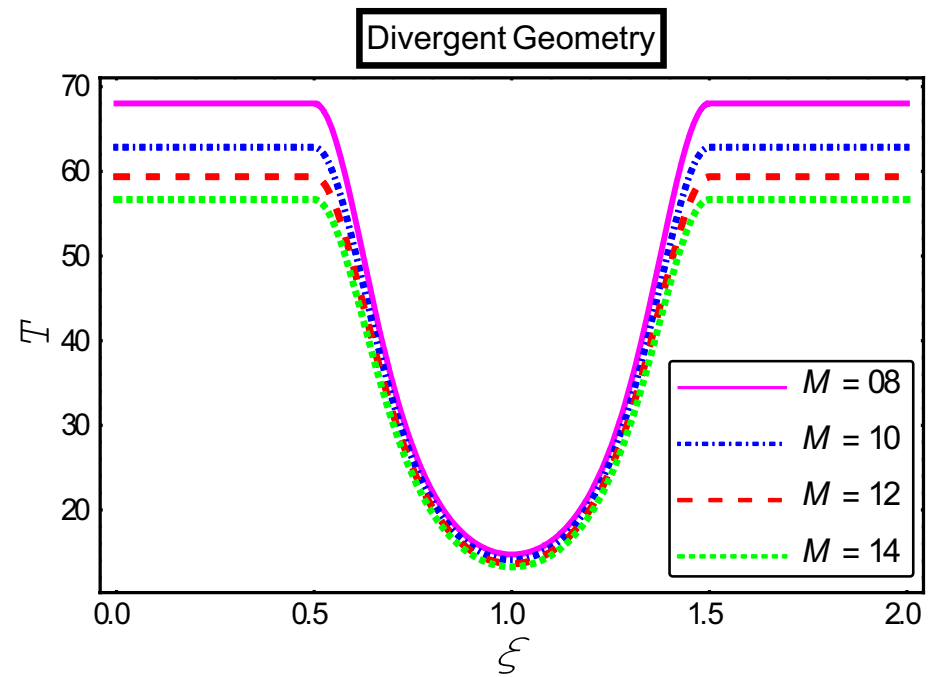

Figure 10. Impact of Hartmann numbers on the temperature profile.

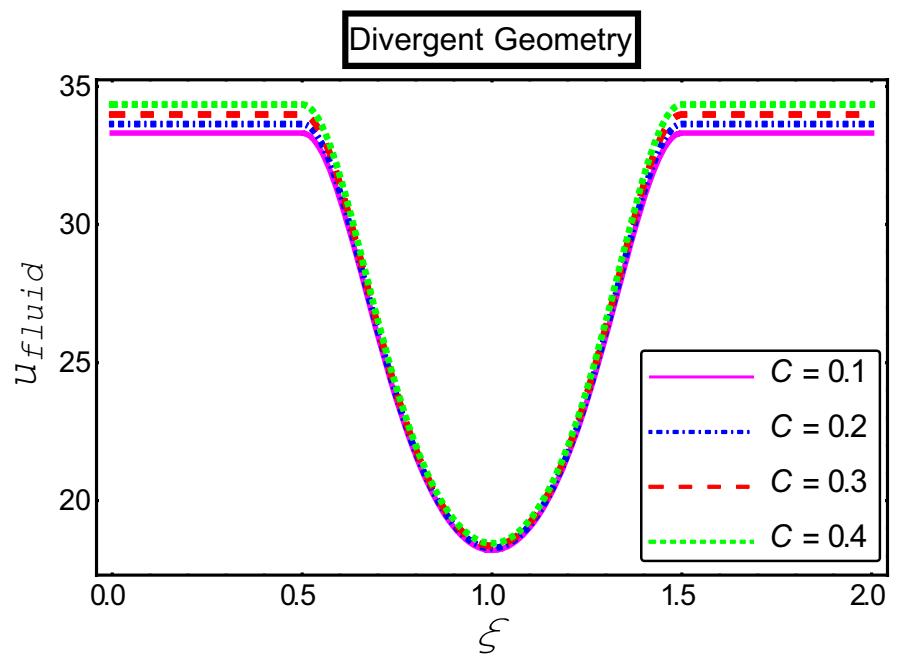

Figure 11. Impact of fluid velocity " $C$ " parameters on fluid velocity.

the impact of magnetic field on electro-osmotic flow Jeffrey fluid. One can notice that a reasonable enhancement in the behavior of fluid and particles velocity is observed as the strong magnetic field is applied. Variation in the number density of gold particles is sketched in Figs. 11, 12 and 13. With the addition of supplementary gold particles, the momentum of both phases gets more aggravated, due to their random motion in the divergent channel. However, the temperature profile shows a decline in Fig. 13. This suggests that heat is being transferred from the region of high temperature to a region of lower temperature. Helmholtz-Smoluchowski velocity $U_{H S}$ is another important emerging parameter. Helmholtz-Smoluchowski velocity has an effective contribution to the flow to determine the volumetric flow rate of viscoelastic fluids in microchannels. The impact of $U_{H S}$ on velocity and temperature profile are shown in Figs. 14, 15 and 16. It is observed that the increasing values of $U_{H S}$ decline the velocity profiles in both fluid and particle phases. However, it can be observed in Fig. 16 variation in $U_{H S}$ supports the temperature profile by increasing the force of friction between the adjacent layers of the base fluid. Finally, the influence of heat conduction from the wall on the viscous fluid is given in Fig. 17. It is noticed that more energy comes into the system due to slow down the process of conduction of heat by viscous dissipation when Brinkman number $B_{r}$ is varied. Hence, the temperature of multiphase flow rises. 


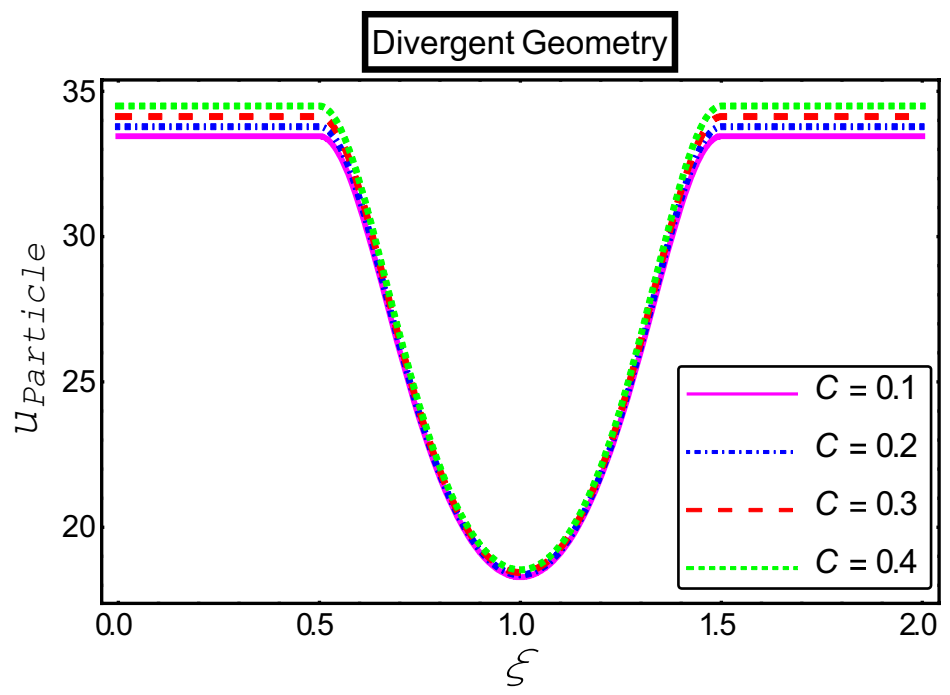

Figure 12. Impact of fluid velocity " $C$ " parameters on particle velocity.

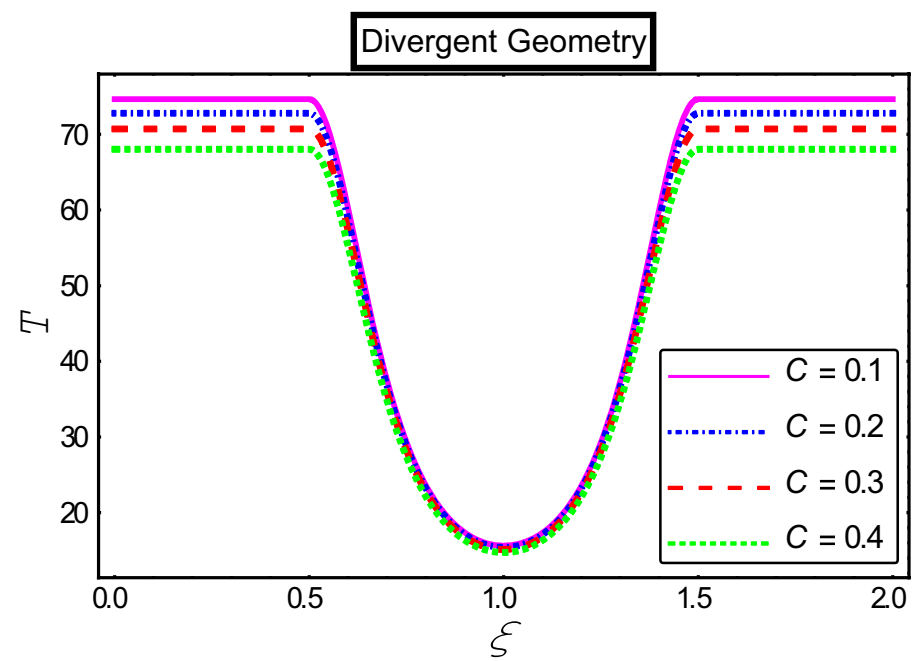

Figure 13. Impact of fluid velocity "C" parameters on the temperature profile.

\section{Concluded remarks}

A closed-form solution is obtained for the heat of a non-Newtonian fluid suspended with gold particles. Electroosmotic multiphase flow is analyzed in a divergent channel under the influence of viscous dissipation and thermal radiation. The most noteworthy observations catalog as:

- Jeffrey parameter corresponds to the rise of both velocity profiles.

- The electro-osmotic parameter $m$ and Helmholtz-Smoluchowski velocity $U_{H S}$ act differently on thermal and momentum distribution.

- Additional gold particles expedite the flow of both phases.

- More energy is added to the system due to Brinkman number $B_{r}$. 


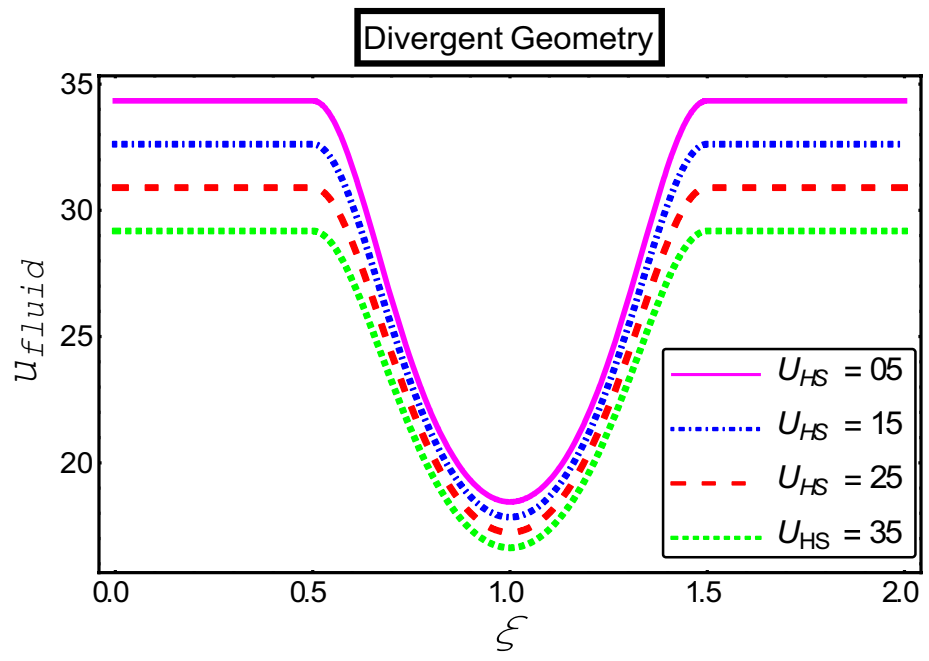

Figure 14. Impact of Helmholtz-Smoluchowski parameter on fluid velocity.

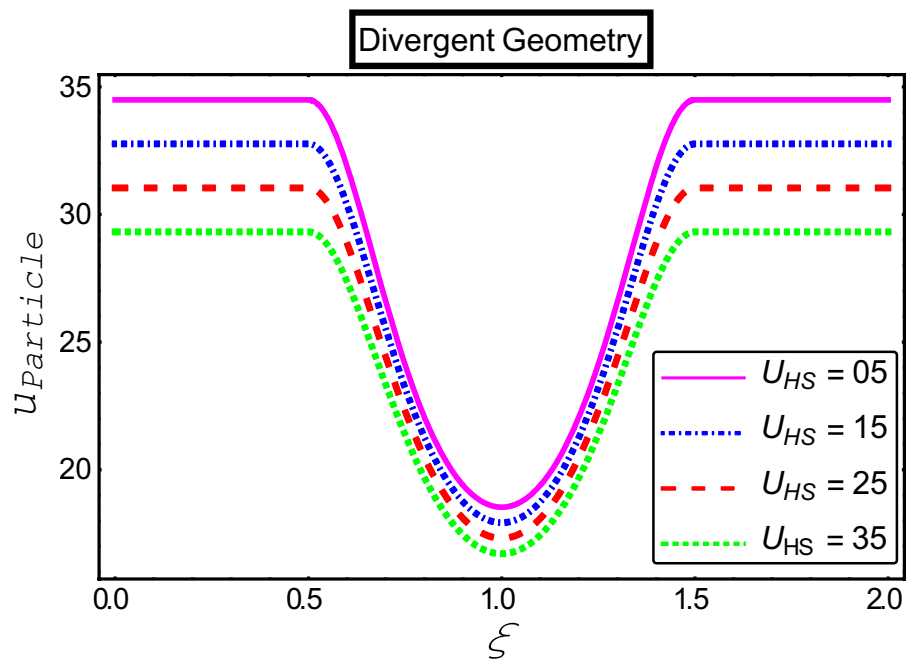

Figure 15. Impact of Helmholtz-Smoluchowski parameter on particle velocity.

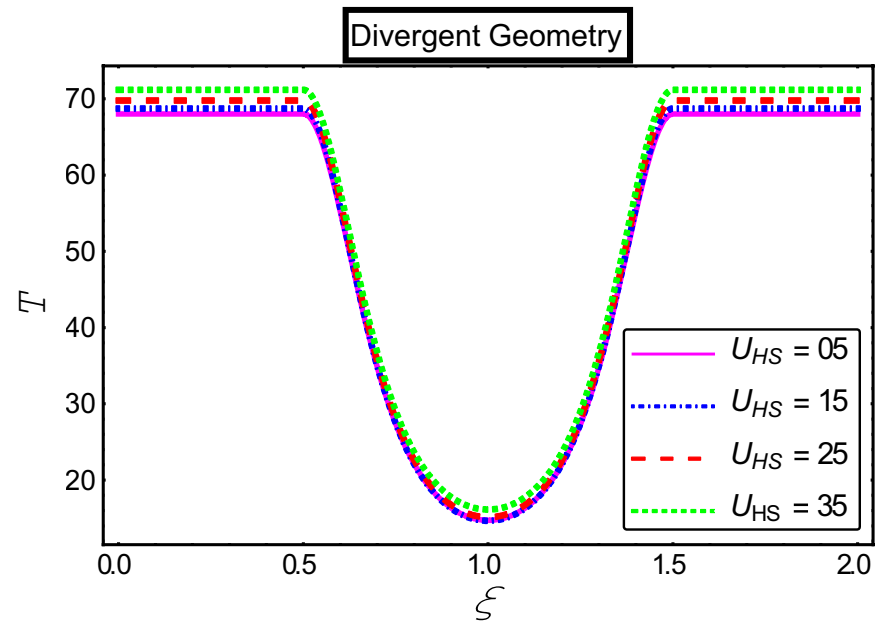

Figure 16. Impact of Helmholtz-Smoluchowski parameter on the temperature profile. 


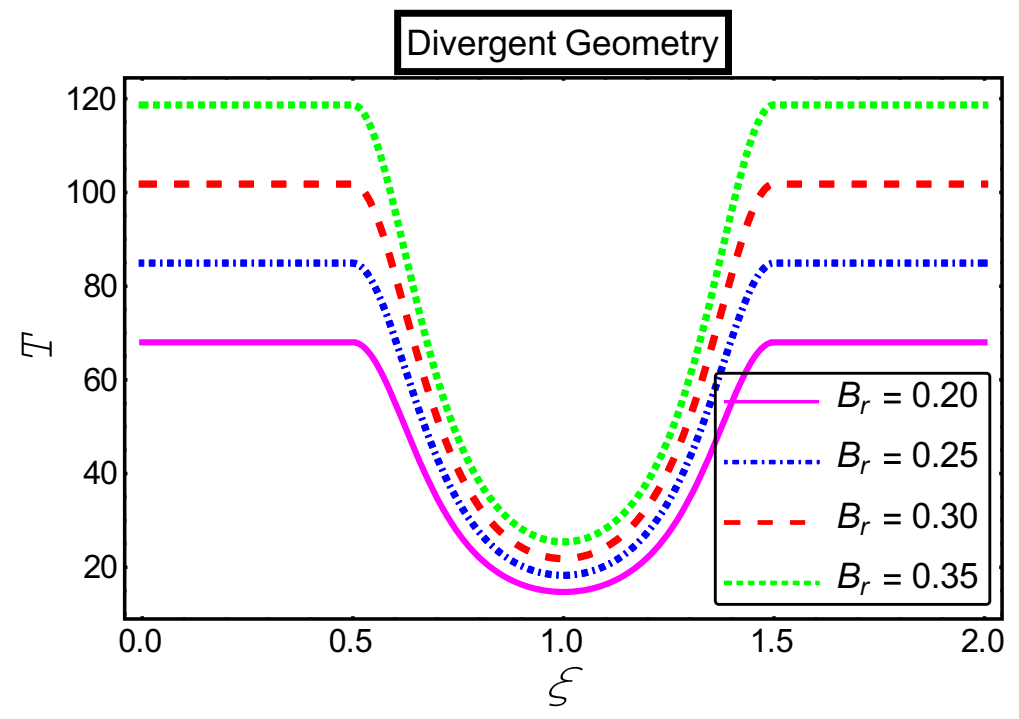

Figure 17. Impact of Brinkman number parameter on the temperature profile.

Received: 25 July 2021; Accepted: 16 November 2021

Published online: 08 December 2021

\section{References}

1. Mekheimer, Kh. S., Hasona, W. M., Abo-Elkhair, R. E. \& Zaher, A. Z. Peristaltic blood flow with gold nanoparticles as a third grade nanofluid in catheter: Application of cancer therapy. Phys. Lett. A 382(3), 85-93 (2018).

2. Abdelsalam, S. I., Mekheimer, Kh. S. \& Zaher, A. Z. Alterations in blood stream by electroosmotic forces of hybrid nanofluid through diseased artery: Aneurysmal/stenosed segment. Chin. J. Phys. 67, 314-329 (2020).

3. Nazeer, M. et al. Effects of radiative heat flux and joule heating on electro-osmotically flow of non-Newtonian fluid: Analytical approach. Int. Commun. Heat Mass Transf. 117, 104744 (2020).

4. Hussain, F., Ellahi, R. \& Zeeshan, A. Mathematical models of electro-magnetohydrodynamic multiphase flows synthesis with nano-sized hafnium particles. Appl. Sci. 8(2), 275 (2018).

5. Ellahi, R. et al. Study of two-phase Newtonian nanofluid flow hybrid with Hafnium particles under the effects of slip. Inventions 5(1), 6 (2020).

6. Mekheimer, K. S., Zaher, A. Z. \& Hasona, W. M. Entropy of AC electro-kinetics for blood mediated gold or copper nanoparticles as a drug agent for thermotherapy of oncology. Chin. J. Phys. 65, 123-138 (2020).

7. Saleem, S., Subia, G. S., Nazeer, M., Hussain, F. \& Hameed, M. K. Theoretical study of electro-osmotic multiphase flow of Jeffrey fluid in a divergent channel with lubricated walls. Int. Commun. Heat Mass Transf. 127, 105548 (2021).

8. Firdous, H., Husnine, S. M., Hussain, F. \& Nazeer, M. Velocity and thermal slip effects on two-phase flow of MHD Jeffrey fluid with the suspension of tiny metallic particles. Phys. Scripta 96(2), 025803 (2020).

9. Rana, P., Shukla, N., Gupta, Y. \& Pop, I. Analytical prediction of multiple solutions for MHD Jeffrey-Hamel flow and heat transfer utilizing KKL nanofluid model. Phys. Lett. A 383(2-3), 176-185 (2019).

10. Ahmad, I., Zafar, H., Kiyani, M. Z. \& Farooq, S. Zero mass flux characteristics in Jeffrey nanoliquid flow by a non-linear stretchable surface with variable thickness. Int. J. Heat Mass Transf. 132, 1166-1175 (2019).

11. Ellahi, R., Hussain, F., Ishtiaq, F. \& Hussain, A. Peristaltic transport of Jeffrey fluid in a rectangular duct through a porous medium under the effect of partial slip: An application to upgrade industrial sieves/filters. Pramana 93(3), 34 (2019).

12. Ellahi, R., Rahman, S. U. \& Nadeem, S. Blood flow of Jeffrey fluid in a catharized tapered artery with the suspension of nanoparticles. Phys. Lett. A 378(40), 2973-2980 (2014).

13. Hussain, F., Nazeer, M., Altanji, M., Saleem, A. \& Ghafar, M. M. Thermal analysis of Casson rheological fluid with gold nanoparticles under the impact of gravitational and magnetic forces. Case Stud. Therm. Eng. 28, 101433 (2021).

14. Nazeer, M. et al. Development of mathematical modeling of multi-phase flow of Casson rheological fluid: Theoretical approach. Chaos Solitons Fractals 150, 111198 (2021).

15. Xiong, P. et al. Two-phase flow of couple stress fluid thermally effected slip boundary conditions: Numerical analysis with variable liquids properties. Alex. Eng. J. https://doi.org/10.1016/j.aej.2021.09.012 (2021).

16. Nazeer, M. et al. Perturbation solution of the multiphase flows of third grade dispersions suspended with Hafnium and crystal particles. Surf. Interfaces 22, 100803 (2021).

17. Nazeer, M., Khan, M. I., Chu, Y., Kadry, S. \& Eid, M. R. Mathematical modeling of multiphase flows of third-grade fluid with lubrication effects through an inclined channel: Analytical treatment. J. Dispersion Sci. Technol. https://doi.org/10.1080/01932691. 2021.1877557 (2021).

18. Ellahi, R. \& Hussain, F. Simultaneous effects of MHD and partial slip on peristaltic flow of Jeffrey fluid in a rectangular duct. J. Magn. Magn. Mater. 393, 284-292 (2015).

19. Ellahi, R., Zeeshan, A., Hussain, F. \& Abbas, T. Study of shiny film coating on multi-fluid flows of a rotating disk suspended with nano-sized silver and gold particles: A comparative analysis. Coatings 8(12), 422 (2018).

20. Ellahi, R., Zeeshan, A., Hussain, F. \& Abbas, T. Two-phase Couette flow of Couple stress fluid with temperature dependent viscosity thermally affected by magnetized moving surface. Symmetry 11(5), 647 (2019).

21. Zeeshan, A., Ellahi, R., Mabood, F. \& Hussain, F. Numerical study on bi-phase coupled stress fluid in the presence of Hafnium and metallic nanoparticles over an inclined plane. Int. J. Numer. Meth. Heat Fluid Flow 29(8), 2854-2869 (2019).

22. Cleary, P. W., Hilton, J. E. \& Sinnott, M. D. Modelling of industrial particle and multiphase flows. Powder Technol. 314, 232-252 (2017). 
23. Zheng, D., Wang, X. \& Yuan, Q. The flow and heat transfer characteristics in a rectangular channel with convergent and divergent slit ribs. Int. J. Heat Mass Transf. 141, 464-475 (2019).

24. Mekheimer, K. S. \& Kot, M. E. Suspension model for blood flow through arterial catheterization. Chem. Eng. Commun. 197(9), 1195-1214 (2010).

25. Bhatti, M. M., Alamri, S. Z., Ellahi, R. \& Abdelsalam, S. I. Intra-uterine particle-fluid motion through a compliant asymmetric tapered channel with heat transfer. J. Therm. Anal. Calorim. https://doi.org/10.1007/s10973-020-10233-9 (2020).

26. Rahman, S. U., Ellahi, R., Nadeem, S. \& ZaighamZia, Q. M. Simultaneous effects of nanoparticles and slip on Jeffrey fluid through tapered artery with mild stenosis. J. Mol. Liq. 218, 484-493 (2016).

27. Abdelsalam, S. I., Velasco-Hernández, J. X. \& Zaher, A. Z. Electro-magnetically modulated self-propulsion of swimming sperms via cervical canal. Biomech. Model. Mechanobiol. 20(3), 861-878 (2021).

28. Bhatti, M. M., Marin, M., Zeeshan, A. \& Abdelsalam, S. I. Editorial: Recent trends in computational fluid dynamics. Front. Phys. 8, $593111(2020)$.

29. AbdElmaboud, Y., Mekheimer, K. S. \& Abdelsalam, S. I. A study of nonlinear variable viscosity in finite-length tube with peristalsis. Appl. Bionics Biomech. 11(Article ID 618637), 10 (2014).

30. Bhatti, M. M. \& Abdelsalam, S. I. Thermodynamic entropy of a magnetized Ree-Eyring particle-fluid motion with irreversibility process: A mathematical paradigm. Zamm-Z. Angew. Math. Mech. 101(6), e202000186 (2020).

31. Raza, R., Mabood, F., Naz, R. \& Abdelsalam, S. I. Thermal transport of radiative Williamson fluid over stretchable curved surface. Therm. Sci. Eng. Progress 23, 100887 (2021).

32. Ge-JiLe, H. et al. Two-phase flow of MHD Jeffrey fluid with the suspension of tiny metallic particles incorporated with viscous dissipation and Porous Medium. Adv. Mech. Eng. 13(3), 1-15 (2021).

33. Nazeer, M. et al. Multi-phase flow of Jeffrey Fluid bounded within magnetized horizontal surface. Surf. Interfaces 22, 100846 (2021).

\section{Acknowledgments}

This research was supported by Basic Science Research Program through the National Research Foundation of Korea (NRF) funded by the Ministry of Education (No. 2017R1D1A1B05030422).

\section{Author contributions}

Y.J.X, M.N. and F.H. prepared the literature survey, references section, constructed analytic solutions and sketched the graphs. M.I.K., M.K.H., N.A.S. and J.D.C. implemented software package and calculated the tabulated values. All the authors have read and approved the final draft of the manuscript.

\section{Competing interests}

The authors declare no competing interests.

\section{Additional information}

Correspondence and requests for materials should be addressed to J.D.C.

Reprints and permissions information is available at www.nature.com/reprints.

Publisher's note Springer Nature remains neutral with regard to jurisdictional claims in published maps and institutional affiliations.

(c) (1) Open Access This article is licensed under a Creative Commons Attribution 4.0 International License, which permits use, sharing, adaptation, distribution and reproduction in any medium or format, as long as you give appropriate credit to the original author(s) and the source, provide a link to the Creative Commons licence, and indicate if changes were made. The images or other third party material in this article are included in the article's Creative Commons licence, unless indicated otherwise in a credit line to the material. If material is not included in the article's Creative Commons licence and your intended use is not permitted by statutory regulation or exceeds the permitted use, you will need to obtain permission directly from the copyright holder. To view a copy of this licence, visit http://creativecommons.org/licenses/by/4.0/.

(C) The Author(s) 2021 